\section{A young patient with concurrent splanchnic dynamic vascular compression syndromes}

\author{
George Salem', Ana I Casanegra², Joshua L Gierman³, \\ Beau M Hawkins ${ }^{2}$ and Alfonso J Tafur ${ }^{2}$
}

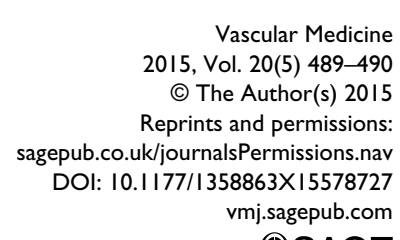

(SAGE

\begin{abstract}
A thirty-year-old lady presented with chronic, postprandial abdominal pain associated with decreased appetite and weight loss. She had a past medical history of ulcerative colitis and past surgical history of Nissen fundoplication, hysterectomy and bilateral oophorectomy for endometriosis. Enhanced computed tomography (CT) of the abdomen/pelvis displayed severe narrowing of the celiac artery (CA) at the level of its ostium (Panel A) with post-stenotic dilatation. In an abdominal vascular ultrasound with respiratory maneuvers we saw a peak systolic velocity (PSV) in the CA during inspiration of $295 \mathrm{~cm} / \mathrm{s}$. The PSV was 108 $\mathrm{cm} / \mathrm{s}$ during expiration for the CA. Peak systolic velocity in the superior mesenteric artery (SMA) was $424 \mathrm{~cm} / \mathrm{s}$ during inspiration. Magnetic resonance angiogram (MRA, Panel B) showed compression of the SMA with post-stenotic dilatation. No signs of perivascular inflammation or fat stranding were appreciated. Serology studies workup showed only weakly positive anti nuclear antibody (ANA) titer. A mesenteric angiography (Panels $C$ and $D$ ) illustrated progression to occlusion of the CA, a dynamic compression of the SMA with kinking mainly during expiration, and post-stenotic dilatation. The pre-operative suspicion for dynamic mesenteric vascular compression syndromes was intraoperatively confirmed with associated scarring along the CA and SMA via the arcuate ligament.
\end{abstract}

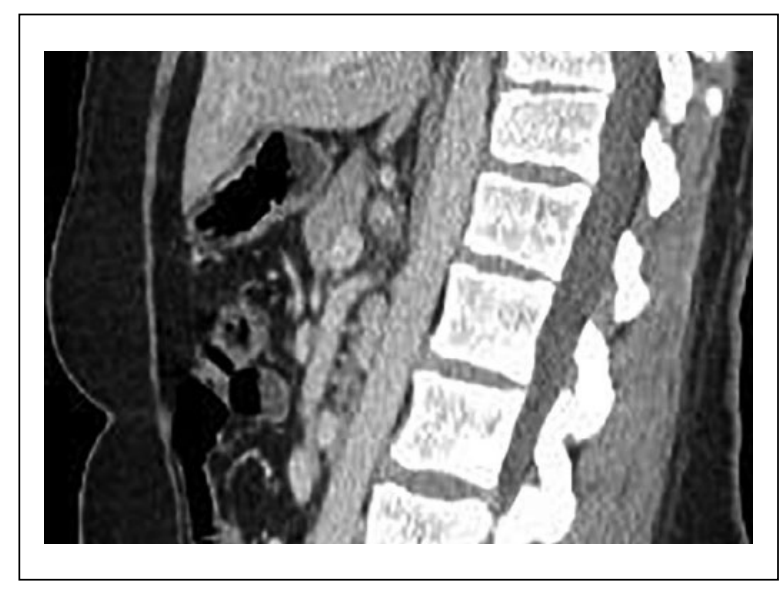

Panel A.

Enhanced computed tomography showing severe narrowing of the celiac artery.

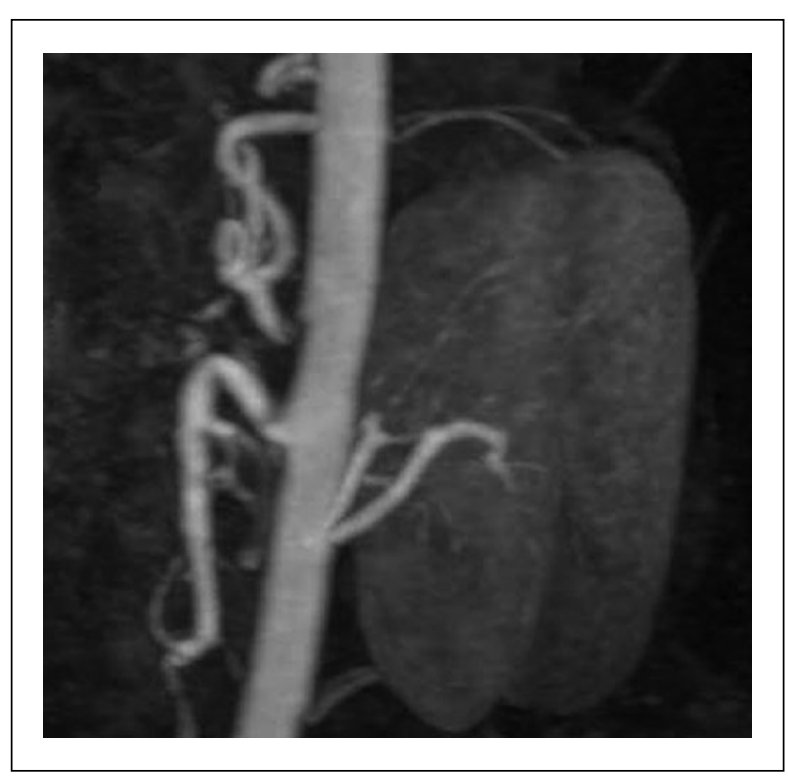

Panel B.

Compression of the superior mesenteric artery on magnetic resonance angiogram (MRA).

Chronic mesenteric ischemia is usually due to atherosclerosis, although it can rarely be caused by fibromuscular dysplasia, segmental arterial mediolysis, or trauma.' Median arcuate ligament syndrome (MALS) is the dynamic compression of the CA by the median arcuate ligament of the diaphragm, typically seen in middle-aged females complaining of postprandial abdominal pain, emesis, and weight loss. The diagnosis is classically made

'Department of Internal Medicine, University of Oklahoma Health Sciences Center, USA

2Department of Medicine, Section of Cardiovascular Medicine, University of Oklahoma Health Sciences Center, USA

${ }^{3}$ Department of Surgery, Section of Vascular Surgery, University of Oklahoma Health Sciences Center, USA

\section{Corresponding author:}

George Salem, Department of Internal Medicine, University of Oklahoma Health Sciences Center, PO Box 2690I, WPI I40, Oklahoma City, OK 73104, USA.

Email: george-salem@ouhsc.edu 


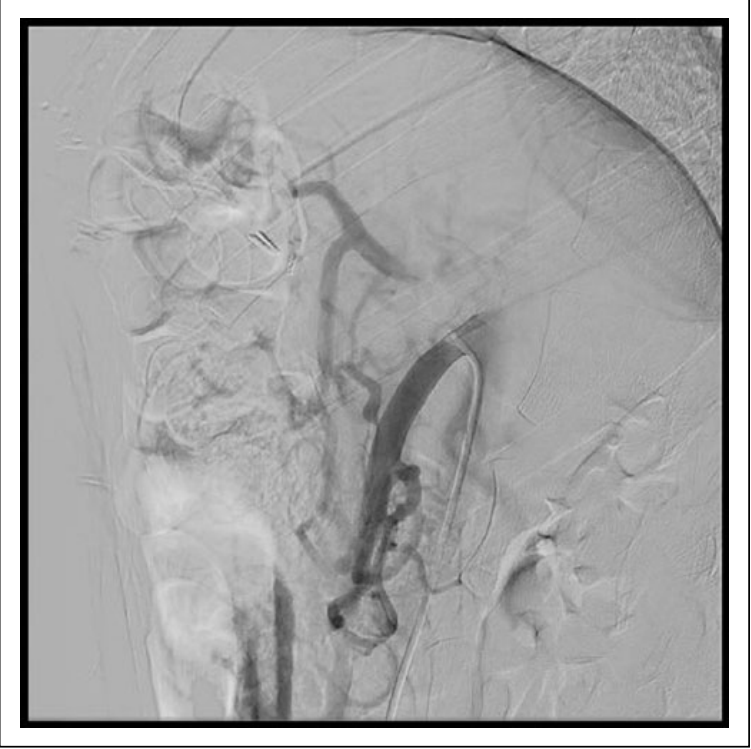

Panel C.

Angiogram with progression to occlusion in the celiac artery.

by angiography, with less invasive diagnostic measures such as Duplex arterial ultrasonography emerging as an important testing modality. As our case confirmed, rarely the CA and SMA can be externally compressed simultaneously in the same patient. ${ }^{2}$

The treatment is surgical division of the median arcuate ligament. ${ }^{3}$ This is curative, although surgical bypass is sometimes necessary. Dynamic vascular compression syndromes are unusual entities. They should be strongly considered as part of the differential diagnosis of chronic mesenteric ischemia without atherosclerosis.

\section{Declaration of conflicting interest}

The authors declare that there is no conflict of interest.

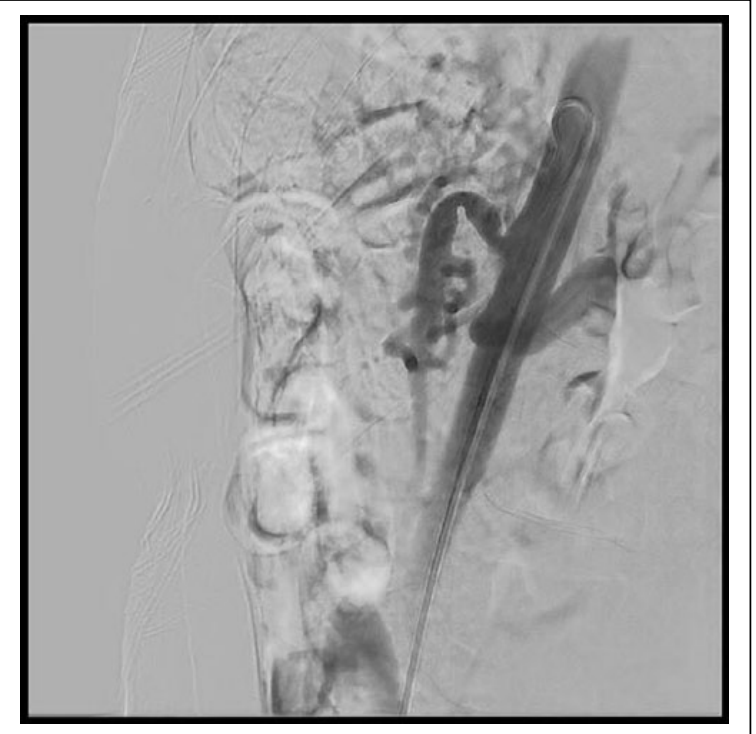

\section{Panel D.}

Kinking and post-stenotic dilatation of the superior mesenteric artery on angiogram.

\section{Funding}

This research received no specific grant from any funding agency in the public, commercial, or not-for-profit sectors.

\section{References}

I. Moawad J and Gewertz BL. Chronic mesenteric ischemia: clinical presentation and diagnosis. Surg Clin North Am 1997; 77: 357-369.

2. Curl JH, Thompson NW and Stanley JC. Median arcuate ligament compression of the celiac and superior mesenteric arteries. Ann Surg 197I; 173(2): 3|4-320.

3. Berard X, Cau J, Déglise S, et al. Laparoscopic surgery for coeliac artery compression syndrome: current management and technical aspects. Eur J Vasc Endovasc Surg 2012; 43(I): 38-42.

'Images in vascular medicine' is a regular feature of Vascular Medicine. Readers may submit original, unpublished images related to clinical vascular medicine. Submissions may be sent to: Heather Gornik, Editor in Chief, Vascular Medicine, via the web-based submission system at http://mc.manuscriptcentral.com/vascular-medicine 